\title{
TRIPLE SEQUENCES AND THEIR LIMITATIONS
}

\author{
C.Gowrishankar, T.Sathish, K.Shalini \\ Assistant Professor \\ Department of Mathematics \\ Government Arts and Science College, Gudalur, The Nilgiris, Tamil Nadu, India - 643212
}

\begin{abstract}
In that paper, we have to established the belief of a sequence and about limits of sequences and the idea of convergence and divergence for a sequence and also it includes the double sequences and triple sequences. We will be developing the principles for triple sequences that is equivalent to the principles of double sequences and single sequence. Also we have an investigate a basic principles for triple sequences and also gives detailed proof of them.
\end{abstract}

Keywords - Single Sequence, Double Sequence, Triple Sequence, Convergence Sequence, Divergence Sequence, Oscillation Sequence, Cauchy Sequence, Triple Subsequence.

\section{INTRODUCTION}

In mathematics, a sequence is specified collection of objects in which repetitions are permitted. Like a set it contains members (elements or items). The number of elements (may be infinite) is called the length of the sequences. Unlike a set, the same elements can appear multiple times at different positions in the sequence.

Formally a sequence can be defined as a function whose domain is either the set of natural numbers (for infinite sequence) or the set of first $\mathrm{n}$ natural numbers (i.e., a sequence of length $n$ ). Sequences are useful in a number of mathematical regulations for studying functions, spaces and other mathematical structures using the convergence properties of sequences. In particular, sequences are basis for the series, which are important to differential equations and analysis. When a symbol has been chosen for denoting a sequence, the $\mathrm{n}^{\text {th }}$ element of the sequence is denoted by $a_{n}$, and this symbol with $\mathrm{n}$ as a subscript.

The elements in a sequence are separated by commas and the length of a sequence is usually denoted by the letter $\mathbf{n}$. The length of a sequence is equal to the number of terms of a sequence is defined by its position in the sequences. For example: $a_{1}, a_{2}, a_{3}, \ldots \ldots, a_{n}$.

In the above, each term is denoted by $\boldsymbol{\alpha}$ with a subscript number denoting its position in the sequence. The first term is $\boldsymbol{\alpha}_{1}$ and the last term is $\boldsymbol{\alpha}_{\boldsymbol{n}}$ where $\mathbf{n}$ is the length of the sequence. Occasionally the first term is also denoted by $\boldsymbol{\alpha}_{\mathbf{0}}$. General formula for computing the $\mathrm{n}^{\text {th }}$ term as a function of $\mathrm{n}$, enclose it in parentheses, and include a subscript indicating the range of values that $\mathrm{n}$ can take. For example, the sequence of even numbers could be written as $(\mathbf{2 n})_{n \in N}$. The variable $\mathrm{n}$ is called an index, and the set of values can take is called an index set.

A triple sequence (say real or complex) can be defined as a function s: $\mathbf{N x N x} \rightarrow R(C)$, where $N, R, C$ denoted by natural numbers, real numbers and complex numbers respectively. The different kinds of notions of triple sequences were established and investigated at the initial stage by Shaniera(2008) and Dutta et.A.J.(2013) and others. Recently Esi.A have established statistical convergence of triple sequences in topological groups.

In that paper we define the belief of triple sequences and its convergency. Also, we have investigated some limitations about triple sequences.

\section{SINGLE SEQUENCE AND DOUBLE SEQUENCE}

\section{DEFINITION 2.1}

A sequence of complex numbers is an infinite ordered list, that is

$$
\left(\mathrm{a}_{\mathrm{n}}\right)_{\mathrm{n}=1}^{\infty}=\left(a_{1}, a_{2,} a_{3_{3}} \ldots . ., a_{n}, \ldots . .\right), a_{n} \in \mathrm{C} \forall n \in N .
$$

\section{DEFINITION 2.2}

A sequence of complex numbers $\left(a_{n}\right)_{n=1}^{\infty}$ is said to converge to $A \in C$ if $\forall \varepsilon>0 \exists$ an $N \in N$ s.t. if $n \geq N$ then $\left|a_{n}-\mathrm{A}\right|<\varepsilon$.

A sequence of complex numbers $\left(a_{n}\right)_{n=1}^{\infty}$ is said to diverge if it does not converge to any $A \in C$.

\section{DEFINITION 2.3}

For each positive integer $\mathrm{n}$, we are given a real number $\boldsymbol{\alpha}_{n}$. Then the list of numbers $a_{1}, a_{2}, a_{3}, \ldots \ldots, a_{n}$ is called a sequence and this ordered list is normally written as

$$
\left(a_{1}, a_{2}, a_{3} \ldots \ldots, a_{n}\right) \text { (or) }\left(a_{n}\right) \text { (or) }\left\{a_{n}\right\} \text {. }
$$

\section{DEFINITION 2.4}

Let $s: N \rightarrow R$ is a sequence and if $a_{n}=s(n)$ for a $n \in N$ then we write the sequence as $\left(a_{n}\right)$ or $\left(a_{1}, a_{2}, \ldots \ldots ..\right)$. A sequence of real numbers is also called real sequence. 


\section{International Journal of Engineering Applied Sciences and Technology, 2021 \\ Vol. 5, Issue 12, ISSN No. 2455-2143, Pages 182-190 \\ Published Online April 2021 in IJEAST (http://www.ijeast.com)}

\section{DEFINITION 2.5}

A sequence $\left(\boldsymbol{\alpha}_{n}\right)$ in $\mathrm{R}$ is said to converge to $\boldsymbol{\alpha}$ be real number if for every $\boldsymbol{\varepsilon}>0, \exists$ positive integer $\mathrm{N}$ (depending on $\boldsymbol{\varepsilon}$ ) s.t. $\left|a_{n}-\boldsymbol{a}\right|<\varepsilon \vee \mathrm{V} n \geq N$.

Also in that case, the number $\boldsymbol{\alpha}$ is called a limit of the sequence $\left(a_{n}\right)$ and $\left(a_{n}\right)$ is called a convergent sequence. A sequence which does not converge is called a divergent sequence.

\section{DEFINITION 2.6}

A double sequence of complex numbers is a function $s: \boldsymbol{N} \times \boldsymbol{N} \rightarrow \boldsymbol{C}$. i.e., we use the symbol $(\boldsymbol{s}(\boldsymbol{n}, \boldsymbol{m}))$ or simply $\left(s_{n, m}\right)$. We say that a double sequence $(s(n, m))$ $\rightarrow a \in \boldsymbol{C}$ and write $\lim _{n, m \rightarrow \infty} s(n, m)=a$, if the given condition is satisfied:

If every $\quad \epsilon>0, \quad \exists \quad N=N(\epsilon) \in N \quad$ s.t. $|s(n, m)-a|<\epsilon \quad \forall n, m \geq N$.

The number $\boldsymbol{a}$ is called double limit of the double sequence $(s(n, m))$. If no such $\boldsymbol{\alpha}$ exists, we say that the sequence $(s(n, m))$ is said to diverges.

\section{DEFINITION 2.7}

If $(s(n, m))$ be double sequence of real numbers.

(i) We say that $(s(n, m)) \rightarrow \infty$, and write $\lim _{n, m \rightarrow \infty} s(n, m)=\infty$, for every $\alpha \in \boldsymbol{R}_{,} \exists$ $K=K(\alpha) \in N \quad$ s.t. $\quad$ if $\quad n, m \geq K_{s} \quad$ then $s(n, m)>\alpha$.

(ii) We say that $(s(n, m)) \rightarrow-\infty$, and we write $\lim _{n, m \rightarrow \infty} s(n, m)=-\infty$, if for every $\beta \in \boldsymbol{R}, \exists$ $K=K(\beta) \in N \quad$ s.t. if $n, m \geq K_{\text {, }}$ then $s(n, m)<\beta$.

We say that $(s(n, m))$ is properly divergent in case we have $\lim _{n, m \rightarrow \infty} s(n, m)=\infty$ or $\lim _{n, m \rightarrow \infty} s(n, m)=-\infty$. In case $(s(n, m))$ does not converge to $a \in \boldsymbol{R}$ and also it does not diverge properly, then we say that $(s(n, m))$ oscillates finitely or infinitely according as $(s(n, m))$ is also bounded or not.

\section{DEFINITION 2.8}

A double sequence $(s(n, m))$ is called bounded if $\exists$ a real number $M>0$ s.t. $|s(n, m)| \leq M, \quad \forall n, m \in N$

\section{DEFINITION 2.9}

For a double sequence $(s(n, m))$, the limits

$\lim _{n \rightarrow \infty}\left(\lim _{m \rightarrow \infty} s(n, m)\right)$, and

$\lim _{m \rightarrow \infty}\left(\lim _{n \rightarrow+\infty} s(n, m)\right)$ are called iterated limits.

\section{DEFINITION 2.10}

A double sequence $(s(n, m))$ of complex numbers is called a Cauchy sequence $\Leftrightarrow$ for every $\epsilon>0, \exists$ a natural number $N=N(\epsilon)$ s.t.

$|s(p, q)-s(n, m)|<\epsilon \forall p \geq n \geq N$ and $q \geq m \geq N$.

\section{DEFINITION 2.11}

Let $(s(n, m))$ be double sequence of real numbers.

(i) If $s(n, m) \geq s(j, k), \forall(n, m) \geq(j, k)$ in $\mathbf{N} \times \boldsymbol{N}$, we say the sequence is decreasing.

(ii) If $s(n, m) \leq s(j, k), \forall(n, m) \leq(j, k)$ in $\mathbf{N} \times \boldsymbol{N}$, we say the sequence is increasing.

(iii) If $(\mathrm{s}(\mathrm{n}, \mathrm{m}))$ is either increasing or decreasing, then we say it is monotone.

\section{DEFINITION 2.12}

Let $(s(n, m))$ be a double sequence of complex numbers and let $\left(k_{1}, r_{1}\right)<\left(k_{2}, r_{2}\right)<\cdots<\left(k_{n}, r_{n}\right)<\cdots$ be strictly increasing sequences of pairs of natural numbers. Then the sequence $\left(\boldsymbol{s}\left(\boldsymbol{k}_{n}, \boldsymbol{r}_{m}\right)\right)$ is called a subsequence of $(s(n, m))$.

\section{TRIPLE SEQUENCE}

The principles of triple sequences is an extension of the single sequence and double sequences. To each triple sequences $s: N \times N \times N \rightarrow C_{\text {s }}$ there corresponds four important limits, namely:
1) $\lim _{l, m, n \rightarrow \infty} s(l, m, n)$
2) $\lim _{l \rightarrow \infty}\left(\lim _{m \rightarrow \infty} \lim _{n \rightarrow \infty} s(l, m, n)\right)$
3) $\lim _{m \rightarrow \infty}\left(\lim _{n \rightarrow \infty} \lim _{l \rightarrow \infty} s(l, m, n)\right)$
4) $\lim _{n \rightarrow \infty}\left(\lim _{l \rightarrow \infty} \lim _{m \rightarrow \infty} s(l, m, n)\right)$

Let $\lim _{l, m, n \rightarrow \infty} s(l, m, n)=a$. Then the iterated limits $\lim _{l \rightarrow \infty}\left(\lim _{m \rightarrow \infty} \lim _{n \rightarrow \infty} s(l, m, n)\right)$, 
$\lim _{m \rightarrow \infty}\left(\lim _{n \rightarrow \infty} \lim _{l \rightarrow \infty} s(l, m, n)\right)$

and

$\lim _{n \rightarrow \infty}\left(\lim _{l \rightarrow \infty} \lim _{m \rightarrow \infty} s(l, m, n)\right)$ exists and both are

equal to $a \Leftrightarrow$

i. $\quad \lim _{l \rightarrow \infty} s(l, m, n)$ exists for each $n, m \in N$

ii. $\quad \lim _{m \rightarrow \infty} s(l, m, n)$ exists for each $l, n \in N$

iii. $\quad \lim _{n \rightarrow \infty} s(l, m, n)$ exists for each $l, m \in N$

\subsection{TRIPLE SEQUENCES AND THEIR LIMITS}

In that section, we have to introduce the triple sequences of complex numbers and also give definition of their convergence, divergence and oscillation.

\section{DEFINITION 3.1}

A triple sequence of complex numbers is a function $s: N \times N \times N \rightarrow C$. We shall use the symbol $(s(l, m, n))$ or simply $\left(s_{l, m, n}\right)$. We say the triple sequence $(s(l, m, n))$ converges to $a \in C$ and we write $\lim _{l, m, n \rightarrow \infty} s(l, m, n)=a$, if the following is satisfied:

For every $\quad \epsilon>0, \quad \exists \quad N=N(\epsilon) \in N \quad$ s.t. $|s(l, m, n)-a|<\epsilon \forall l, m, n \geq N$.

The number $a$ is called the triple limit of the triple sequence $(s(l, m, n))$. If no such $a$ exists, we say that the sequence $(s(l, m, n))$ is diverges.

\section{DEFINITION 3.2}

If $(s(l, m, n))$ be triple sequence of real numbers.

i. We say $(s(l, m, n)) \rightarrow \infty$, and we write $\lim _{l, m, n \rightarrow \infty} s(l, m, n)=\infty$, if for every $\alpha \in \boldsymbol{R} \exists$ $K=K(\alpha) \in \boldsymbol{N} \quad$ s.t. $\quad$ if $\quad l, m, n \geq K \quad$ then $s(l, m, n)>\alpha$.

ii. We say $s(l, m, n) \rightarrow-\infty$, and we write $\lim _{l, m, n \rightarrow \infty} s(l, m, n)=-\infty$, if for every $\beta \in \boldsymbol{R}$ $\exists K=K(\beta) \in N$ s.t. if $l, m, n \geq K$ then $s(l, m, n)<\beta$.

We say $(s(l, m, n))$ is properly divergent in case we have $\lim _{l, m, n \rightarrow \infty} s(l, m, n)=\infty$ or $\lim _{l, m, n \rightarrow \infty} s(l, m, n)=-\infty$. In case $(s(l, m, n))$ does not converge to $a \in \boldsymbol{R}$ and also it does not diverge properly, then we say that $(s(l, m, n))$ oscillates finitely or infinitely according as $(s(l, m, n))$ is also bounded or not.
For example, the sequence $\left((-1)^{l+m+n}\right)$ oscillates finitely, while the sequence $\left((-1)^{l+m+n}(l+m+n)\right)$ oscillates infinitely.

\section{EXAMPLE}

(a) If the triple sequence $s(l, m, n)=\frac{1}{l+m+n}$, we have

$$
\lim _{l, m, n \rightarrow \infty} s(l, m, n)=0
$$

To see this, given $\epsilon>0$, choose $N \in N$ s.t. $N>\frac{3}{\epsilon}$.

Then $\forall l, m, n \geq N$, we have $\frac{1}{i}, \frac{1}{m}, \frac{1}{n} \leq \frac{1}{N}$, which implies that

$|s(l, m, n)-0|=\left|\frac{1}{l+m+n}\right|<\frac{1}{l}$

$+\frac{1}{m}+\frac{1}{n}<\frac{1}{N}+\frac{1}{N}+\frac{1}{N}=\frac{3}{N}<\epsilon$

$\therefore s(l, m, n)$ is convergent.

(b) The triple sequence $s(l, m, n)=\frac{l}{l+m+n}$ is divergent. Indeed, $\quad \mathrm{V}$ sufficiently large $l, m, n \in N$ with $l=m=n, \quad$ then $\quad s(l, m, n)=\frac{1}{3}, \quad$ whereas $\quad \mathrm{V}$ sufficiently large $l, m, n \in N$ with $l=m=2 n$, we have $s(l, m, n)=\frac{2}{5}$. It follows that $s(l, m, n)$ does not converge to a for any $a \in \boldsymbol{R}$ as $l, m, n \rightarrow \infty$.

(c) The triple sequence $s(l, m, n)=l+m+n$ is properly divergent to $\infty$. Indeed, given $\alpha \in R, \exists \mathrm{K} \in \boldsymbol{N}$ s.t. $K>\alpha$. Then $l, m, n \geq K \Rightarrow l+m+n>\alpha$.

(d) The triple sequence $s(l, m, n)=1-l-m-n$ is properly divergent to $-\infty$. Indeed, given $\beta \in R, \exists \mathrm{K}$ $\in N$ s.t. $K>-\frac{\beta}{3}+\frac{1}{3}$. Then $l, m, n \geq K$

$\Rightarrow-l,-m,-n<\frac{\beta}{3}-\frac{1}{3} \Rightarrow 1-l-m-n<\beta$.

\section{THEOREM 3.1 (Uniqueness of triple limits)}

A triple sequence of complex numbers can have at most one limit.

\section{PROOF}

Let $a, a^{\prime}, a^{n}$ are both limits $(s(l, m, n))$. Then given $\epsilon>0 \exists$ a natural number $N_{1}, N_{2}$ and $N_{3}$ s.t.

$$
l, m, n \geq N_{1} \Rightarrow|s(l, m, n)-a|<\frac{E}{4}
$$


$l, m, n \geq N_{2} \Rightarrow\left|s(l, m, n)-a^{\prime}\right|<\frac{\epsilon}{4}$

and s.t.

$$
l, m, n \geq N_{3} \Rightarrow\left|s(l, m, n)-a^{\prime \prime}\right|<\frac{\varepsilon}{4}
$$

Let $\quad N:=\max \left\{N_{1}, N_{2}, N_{3}\right\} . \quad$ Then $\quad \mathrm{V} \quad l, m, n \geq N$ implication (1), (2) and (3) yield,

0

$\left.\leq\left|a-a^{\prime \prime}\right|=\mid a-s(l, m, n)+s(l, m, n)-a^{\prime}+a^{\prime}+s(l, m, n)-s(l, m, n)-a^{\prime \prime}\right) \mid$

$\leq|s(l, m, n)-a|+\left|s(l, m, n)-a^{\prime}\right|+\left|s(l, m, n)-a^{\prime}\right|+\left|s(l, m, n)-a^{\prime \prime}\right|$

$$
<\frac{E}{4}+\frac{E}{4}+\frac{E}{4}+\frac{E}{4}=\frac{4 E}{4}=\epsilon,
$$

$0 \leq\left|a-a^{n}\right|<\epsilon$

It follows that $a-a^{\prime \prime}=0$. Hence the limit is unique.

\section{DEFINITION 3.3}

A triple sequence $(s(l, m, n))$ is called bounded if $\exists$ a real number $M>0$ s.t. $|s(l, m, n)| \leq M, \forall l, m, n \in N$

\section{THEOREM 3.2}

The convergent triple sequence of complex numbers is bounded.

\section{PROOF}

Given $s(l, m, n)$ is convergent triple sequence.

Suppose $s(l, m, n) \rightarrow a$ and let $\epsilon=1$. Then $\exists N \in N_{\text {s.t. }}$. $l, m, n \geq N \Rightarrow|s(l, m, n)-a|<1$

Then the triangle inequality yield that, $|s(l, m, n)|<1+|a| \forall l, m, n \geq N$.

Let

$M:=\max$

$\{(|s(1,1,1)|,|s(1,1,3)|,|s(1,3,1)|,|s(3,1,1)|, \ldots \ldots,|s(N-1, N-1, N-1)|$,

Clearly, $|s(l, m, n)| \leq M \quad \forall l, m, n \in N$.

$\therefore$ The given sequence is bounded.

\section{DEFINITION 3.4}

For a triple sequence $(s(l, m, n))$ the limits,

$\lim _{m \rightarrow \infty} \lim _{n \rightarrow \infty}\left(\lim _{l \rightarrow \infty} s(l, m, n)\right), \lim _{l \rightarrow \infty} \lim _{n \rightarrow \infty}\left(\lim _{m \rightarrow+\infty} s(l, m, n)\right)$ and $\lim _{l \rightarrow \infty} \lim _{m \rightarrow \infty}\left(\lim _{n \rightarrow \infty} s(l, m, n)\right) \quad$ are called iterated limits.

\section{EXAMPLE}

Consider the sequence $s(l, m, n)=\frac{l}{l+m+n}$.

Then for every $m, n \in N, \lim _{l \rightarrow \infty} s(l, m, n)=1$

$\Rightarrow \lim _{m, n \rightarrow \infty}\left(\lim _{l \rightarrow \infty} s(l, m, n)\right)=1$

For every $l, n \in N, \lim _{m \rightarrow \infty} s(l, m, n)=0$

$\Rightarrow \lim _{l, n \rightarrow \infty}\left(\lim _{m \rightarrow \infty} s(l, m, n)\right)=0$

For every $l, m \in N, \lim _{n \rightarrow \infty} s(l, m, n)=0$

$\Rightarrow \lim _{l, m \rightarrow \infty}\left(\lim _{n \rightarrow \infty} s(l, m, n)\right)=0$

Therefore, the triple limit of this sequence does not exist.

\section{THEOREM 3.3}

Let $\quad \lim _{l, m, n \rightarrow \infty} s(l, m, n)=a$. Then

$\lim _{m, n \rightarrow \infty}\left(\lim _{l \rightarrow \infty} s(l, m, n)\right)=a \Leftrightarrow$

$\lim _{l \rightarrow \infty} s(l, m, n)$ exists for each $m, n \in N$.

PROOF

The necessity is obviously true. For sufficiency, assume that $\lim _{l \rightarrow \infty} s(l, m, n)=c_{l}$ for each $m, n \in N$. To show that

$$
c_{l} \rightarrow a \text { as } l \rightarrow \infty \text {. }
$$

Let $\in>0$ be given.

Since $s(l, m, n) \rightarrow a$ as $l, m, n \rightarrow \infty, \exists N_{1} \in \boldsymbol{N}$ s.t.

$l, m, n \geq N_{1} \Rightarrow|s(l, m, n)-a|<\frac{\epsilon}{4}$

Since for each $m \in N, s(l, m, n) \rightarrow c_{m}$ as $n \rightarrow \infty, \exists$

$N_{2} \in N$ s.t.

$n \geq N_{2} \quad \Rightarrow\left|s(l, m, n)-c_{m}\right|<\frac{\epsilon}{4}$

Similarly, for each $n \in N, s(l, m, n) \rightarrow c_{n}$ as $m \rightarrow \infty, \exists$ $N_{3} \in N_{\text {s.t. }}$

$m \geq N_{3} \quad \Rightarrow\left|s(l, m, n)-c_{n}\right|<\frac{E}{4}$

Now choose $m, n \geq \max \left\{N_{1}, N_{2}, N_{3}\right\}$. Then $\forall l \geq N_{1}$, we have

$\left|c_{l}-a\right|=\left|c_{l}-s(l, m, n)+s(l, m, n)-c_{m}+c_{m}-s(l, m, n)+s(l, m, n)-a\right|$ $\leq\left|c_{l}-s(l, m, n)\right|+\left|s(l, m, n)-c_{m}\right|$ $+\left|c_{m}-s(l, m, n)\right|+|s(l, m, n)-a|$ $<\frac{E}{4}+\frac{E}{4}+\frac{E}{4}+\frac{E}{4}=\epsilon$

Hence, $c_{l} \rightarrow a$ as $l \rightarrow \infty$.

\subsection{CAUCHY TRIPLE SEQUENCES} DEFINITION 3.5 


\section{International Journal of Engineering Applied Sciences and Technology, 2021 \\ Vol. 5, Issue 12, ISSN No. 2455-2143, Pages 182-190 \\ Published Online April 2021 in IJEAST (http://www.ijeast.com)}

A triple sequence $(s(l, m, n))$ of complex numbers is called a Cauchy sequence $\Leftrightarrow$ for every $\epsilon>0, \exists$ a natural number $N=N(\epsilon)$ s.t.

$|s(p, q, r)-s(l, m, n)|<\epsilon \forall p \geq l \geq N, q \geq m \geq N$ and $r \geq n \geq N$.

\section{THEOREM 3.4}

(Cauchy Convergence Criterion for Triple Sequences)

A triple sequence $(s(l, m \cdot n))$ of complex numbers

converges $\Leftrightarrow$ it is a Cauchy sequence.

\section{PROOF}

$(\Rightarrow)$ Assume $s(l, m, n) \rightarrow a$ as $l, m, n \rightarrow \infty$.

Given $\epsilon>0, \exists N \in N$ s.t.

$|s(l, m, n)-a|<\frac{\epsilon}{2} \forall l, m, n \geq N$.

Hence $\quad \forall p \geq l \geq N, \quad \forall q \geq m \geq N$

and

$\forall r \geq n \geq N$, we have

$|s(p, q, r)-s(l, m, n)|=|s(p, q, r)-a+a-s(l, m, n)|$

$$
\begin{aligned}
& \leq|s(p, q, r)-a|+|s(l, m, n)-a| \\
& <\frac{E}{2}+\frac{E}{2}=\epsilon
\end{aligned}
$$

i.e., $(s(l, m, n))$ a Cauchy sequence.

$(<=)$ Next, we assume that $(s(l, m, n))$ a Cauchy sequence, and let $\epsilon>0$ be given.

Taking $l=m=n$ and writing $s(l, m, n)=b_{n}$, we see that $\exists k \in N$ s.t. $\left|b_{p}-b_{n}\right|<\epsilon \quad \forall p \geq n \geq \boldsymbol{K}$

$\therefore$ By Cauchy's Criterion for single sequence, the sequence $\left(b_{n}\right)$ converges to $a \in C$.

Hence $\exists N_{1} \in \boldsymbol{N}$ s.t.

$\left|b_{n}-a\right|<\frac{E}{2} \quad \forall n \geq N_{1}$

Since $(s(l, m, n))$ a Cauchy sequence, $\exists N_{2} \in N_{\text {s.t. }}$.

$\left|s(p, q, r)-b_{n}\right|<\frac{E}{2} \quad \forall p, q, r \geq n \geq N_{2}$

Let $N:=\max \left\{N_{1}, N_{2}\right\}$ and choose $n \geq N$. Then, by $(l)$ and (2), we have

$$
\begin{aligned}
|s(p, q, r)-a| & \leq\left|s(p, q, r)-b_{n}\right|+\left|b_{n}-a\right| \\
& <\frac{\epsilon}{2}+\frac{\varepsilon}{2}=\epsilon \quad \forall p, q, r \geq N .
\end{aligned}
$$

Hence, $(s(l, m, n))$ converges to $a$.

\subsection{TRIPLE SUBSEQUENCES}

In that section, we have to study triple subsequences and prove some results about their convergence and its relation to the convergence of the original triple sequence.

\section{DEFINITION 3.6}

If $(s(l, m, n))$ be triple sequence of complex numbers and let

$\left(k_{1}, r_{1}, t_{1}\right)<\left(k_{2}, r_{2}, t_{2}\right)<\ldots \ldots<\left(k_{n}, r_{n}, t_{n}\right)<$

be strictly increasing sequences of pairs of natural numbers.

Then the sequence $\left(s\left(k_{l}, r_{m}, t_{n}\right)\right)$ is called a subsequence

of $(s(l, m, n))$.

\section{THEOREM 3.5}

If a triple sequence $(s(l, m, n))$ of complex numbers converge to a complex number $a$, then any subsequence of $(s(l, m, n))$ also converge to $a$.

\section{PROOF}

Let $\left(s\left(k_{l}, r_{m}, t_{n}\right)\right)$ be subsequence of $(s(l, m, n))$ and let $\epsilon>0$ be given.

Then $\exists N=N(\epsilon) \in \mathbf{N}$ s.t.

$$
f, g, h \geq N \Rightarrow|s(f, g, h)-a|<\epsilon
$$

Since

$k_{1} \leq k_{2} \leq \ldots \leq k_{n} \leq \ldots \ldots, r_{1} \leq r_{2} \leq \cdots \leq r_{n} \leq \ldots \ldots$, and $\quad t_{1} \leq t_{2} \leq \ldots \leq t_{n} \leq \ldots \ldots$ we have $k_{l} \geq l, r_{m} \geq m, t_{n} \geq n \quad \forall l, m, n \in N$.

Hence, it follows that

$$
l, m, n \geq N \Rightarrow k_{l}, r_{m}, t_{n} \geq N
$$

$\Rightarrow\left|s\left(k_{l}, r_{m}, t_{n}\right)\right|<\epsilon$

$\therefore \lim _{l, m, n \rightarrow \infty} s\left(k_{l}, r_{m}, t_{n}\right)=a$

\section{THEOREM 3.6 (Divergence Criterion)}

Let $(s(l, m, n))$ be triple sequence of complex numbers. Then the following are equivalent:

(i) The sequence $(s(l, m, n))$ does not converge to $a \in C$

(ii) $\exists$ an $\epsilon_{0}>0$ s.t. for any $k \in N, \exists$ $l_{k}, m_{k}, n_{k} \in N_{\text {s.t. }}$.

$$
l_{k}, m_{k}, n_{k} \geq k \text { and }\left|s\left(l_{k}, m_{k}, n_{k}\right)-a\right| \geq \epsilon_{0} .
$$

(iii) $\exists$ an $\epsilon_{0}>0$ and a subsequence $\left(s\left(l_{k}, m_{k}, n_{k}\right)\right)$ of $(s(l, m, n))$ s.t. 


$$
\left|s\left(l_{k}, m_{k_{z}} n_{k}\right)-a\right| \geq \epsilon_{0}, \forall k \in N
$$

\section{PROOF}

To Prove: $(i) \Rightarrow$ (ii)

If $(s(l, m, n))$ does not converge to $a$, then $\exists \epsilon_{0}>0$ s.t.

$\forall k \in N$, the statement

$$
l, m, n \geq k \Rightarrow|s(l, m, n)-a|<\epsilon_{0} \text { is false }
$$

(i.e) $\forall k \in N$ there are natural numbers

$l_{k}, m_{k}, n_{k} \geq k_{\text {s.t. }}$

$\left|s\left(l_{k}, m_{k_{s}} n_{k}\right)-a\right| \geq \epsilon_{0}$.

To Prove: (ii) $\Rightarrow$ (iii)

Let $\epsilon_{0}$ be as in (ii) and let $l_{1}, m_{1}, n_{1} \in N$ s.t. $l_{1}, m_{1}, n_{1} \geq 1$ and $\left|s\left(l_{1}, m_{1}, n_{1}\right)-a\right| \geq \epsilon_{0}$

Now let $l_{2}, m_{2}, n_{2} \in \boldsymbol{N}$ be s.t.

$l_{2} \geq l_{1}+1, m_{2} \geq m_{1}+1, n_{2} \geq n_{1}+1$ and

$\left|s\left(l_{2}, m_{2}, n_{2}\right)-a\right| \geq \epsilon_{0}$

Let $l_{3}, m_{3}, n_{3} \in \boldsymbol{N}$ be s.t.

$l_{3} \geq l_{2}+1, m_{3} \geq m_{2}+1, n_{3} \geq n_{2}+1$ and

$\left|s\left(l_{3}, m_{3}, n_{3}\right)-a\right| \geq \epsilon_{0}$

Continuing this process, we obtain a subsequence

$\left(s\left(l_{k}, m_{k}, n_{k}\right)\right)$ of $(s(l, m, n))$ s.t.

$\left|s\left(l_{k}, m_{k}, n_{k}\right)-a\right| \geq \epsilon_{0}, \forall k \in N$

To Prove: (iii) $\Rightarrow$ (i)

Suppose $\exists$ an $\epsilon_{0}>0$ and a subsequence $\left(s\left(l_{k,} m_{k}, n_{k}\right)\right)$ of $(s(l, m, n))$ s.t.

\section{$\left.\mid s\left(l_{k}, m_{k}, n_{k}\right)-a\right) \mid \geq \epsilon_{0} \quad \forall k \in N$}

Then $(s(l, m, n))$ cannot converges to a. For if $(s(l, m, n)) \rightarrow a$ then by using theorem "If a triple sequence $(s(l, m, n))$ of complex numbers converges to a complex number $a$, then any subsequence of $(s(l, m, n))$ also converges to $a$ ". The subsequence $\left(s\left(l_{k,}, m_{k}, n_{k}\right)\right)$ would converge to a, which is impossible.

Therefore, the sequence $(s(l, m, n))$ does not converge to $a \in \boldsymbol{C}$.

\section{THEOREM 3.7}

Let $(s(l, m, n))$ be a bounded triple sequence of complex numbers and let $a \in \boldsymbol{C}$ have property that every convergent subsequence of $(s(l, m, n))$ converge to a. Then the sequence $(s(l, m, n))$ converge a.

PROOF
Assume, on the contrary, that the sequence $(s(l, m, n))$ does not converge to $\mathbf{a}$.

Then, by Divergence Theorem 3.6, there exist $\epsilon_{0}>0$ and a subsequence $\left(s\left(l_{k}, m_{k}, n_{k}\right)\right)$ of $(s(l, m, n))$ s.t.

$$
\left|s\left(l_{k}, m_{k}, n_{k}\right)-a\right| \geq \epsilon_{0} \quad \forall k \in N
$$

Since the sequence $(s(l, m, n))$ is bounded, then so is the subsequence $\left(s\left(l_{k,}, m_{k}, n_{k}\right)\right)$.

It comes from the Bolzano-Weierstrass Theorem that $\left(s\left(l_{k}, m_{k}, n_{k}\right)\right)$ has a convergent subsequence, say $\left(s\left(l_{p}, m_{q}, n_{r}\right)\right)$.

Hence, by hypothesis, $\lim _{p, q_{r} r \rightarrow \infty}\left(s\left(l_{p}, m_{q}, n_{r}\right)\right)=a$.

This means that $\exists N=N\left(\epsilon_{0}\right)$ s.t.

$$
\left|\left(s\left(l_{p}, m_{q}, n_{r}\right)\right)-a\right| \geq \epsilon_{0} \quad \forall p, q, r \geq N
$$

Since every term of $\left(s\left(l_{p}, m_{q}, n_{r}\right)\right)$ is also a term of $\left(s\left(l_{k}, m_{k}, n_{k}\right)\right)$, we see that (1) gives a contradiction to (2).

\subsection{ROUGH STATISTICAL CONVERGENCE ON TRIPLE SEQUENCE}

The concept of statistical convergence was established by 'steinhaus' and also independently by Fast for real or complex sequences. Statistical convergence is a generalization concept of convergence, which equivalent to the principles of ordinary convergence.

\section{DEFINITION 3.7}

A triple sequence $s=\left(s_{l, m, n}\right)$ or $s(l, m, n)$ is said to statistically convergent to $0 \in R$, written as $s t-\lim s=0$, provided that the set $\left\{(l, m, n) \in \boldsymbol{N}^{3}:|s(l, m, n)-0| \geq \epsilon\right\}$ has natural density zero for any $\epsilon>0$. In this case, 0 is called statistical limit of the triple sequence $\mathrm{x}$.

If the triple sequence is statistically convergent, then for every $\Theta>0$, infinitely many terms of the sequence may remain outside the $\epsilon$-neighbourhood of the statistical limit, provided that the natural density of the set consisting of the indices of these terms is zero. This is an important property that distinguishes statistical convergence from ordinary convergence. Because the natural density of a finite set is zero, we say that every ordinary convergent sequence is statistically convergent.

\section{DEFINITION 3.8}




\section{International Journal of Engineering Applied Sciences and Technology, 2021 \\ Vol. 5, Issue 12, ISSN No. 2455-2143, Pages 182-190 \\ Published Online April 2021 in IJEAST (http://www.ijeast.com)}

A triple sequence $s=(s(l, m, n))$ is said to statistically analytic if $\exists$ a positive number $M$ s.t. $\delta\left(\left\{(l, m, n) \in N^{3}:|(s(l, m, n))|^{1 / n+m+n} \geq M\right\}\right)=0$

\section{DEFINITION 3.9}

A triple sequence $s(l, m, n)$ is said to be rough convergent(rconvergent) to $k$ denoted as $s(l, m, n) \rightarrow^{r} k_{p}$ provided that $\forall \in>0 \exists i_{E} \in N$ s.t.

$\forall \in>0, \exists i_{\mathrm{E}} \in N: l, m, n \geq i_{\mathrm{E}} \Rightarrow|s(l, m, n)-k|<r+\epsilon$, Or equivalently, if $\lim \sup |s(l, m, n)-k| \leq r$ Here $r$ is called the roughness of degree. If we take $r=0$, then we obtain the ordinary convergence of a triple sequence.

\section{DEFINITION 3.10}

A triple sequence $s(l, m, n)$ is said to $r$ - convergent if $L I M^{r} s \neq \emptyset$.

In this case $r$ is called the convergence degree of the triple sequence $s(l, m, n)$. For $r=0$, we get the ordinary convergence.

\section{THEOREM 3.8}

Let $r>0$. Then a triple sequence $s(l, m, n)$ is $\mathrm{r}$ statistically convergent to $\mathrm{k} \Leftrightarrow \exists$ a triple sequence $p(l, m, n)$ s.t.

$s t-\lim p=k$ and $|s(l, m, n)-p(l, m, n)| \leq r$ for each $(l, m, n) \in \boldsymbol{N}^{3}$.

\section{PROOF}

Necessity: Assume $s(l, m, n) \rightarrow^{r s t} k$. Then,

$s t-\lim \sup |s(l, m, n)-k| \leq r$

Define

$p(l, m, n)=\left\{\begin{array}{cc}k, \quad \text { if }|s(l, m, n)-l| \leq r \\ s(l, m, n)+r\left(\frac{k-s(l, m, n)}{|s(l, m, n)-l|}\right), \text { otherwise }\end{array}\right.$

Then write,

Then write,
$|p(l, m, n)-k|=\left\{\begin{array}{c}\left.|k-k|, \quad \text { if }|s(l, m, n)-l| \leq r^{\prime}=\sup |(s(l, m, n))| 1 / n+m+n \mid:(l, m, n) \in K^{c}\right\}, \\ |s(l, m, n)-k|+r\left(\frac{|k-k|-\mid s(l, m, n)-k]}{|s(l m, n)-k|}\right), \quad \text { otherwisk }=\left\{(l, m, n) \in N^{3}:|(s(l, m, n))|^{1 / l+m+n \geq M\}}\right.\end{array}\right.$

$|p(l, m, n)-k|=\left\{\begin{array}{cc}0, \quad \text { if }|s(l, m, n)-k| \leq r \quad \text { So we have } s t-L I M^{r} s \neq \emptyset . \\ |s(l, m, n)-k|-r\left(\left|\frac{s(h, m, n)-k}{s(l, m, n)-k}\right|\right), \quad \text { otherwise } s t-L I M^{r} s \neq \emptyset \text { for some } r \geq 0,-L M^{r} s\end{array}\right.$ i.e.,
$|p(l, m, n)-k|=\left\{\begin{array}{cc}0, & \text { if }|s(l, m, n)-k| \leq r \\ |s(l, m, n)-k|-r, & \text { otherwise }\end{array}\right.$

We have

$|p(l, m, n)-k| \geq|s(l, m, n)-k|-r$

$\Rightarrow \quad|s(l, m, n)-k-p(l, m, n)+k| \leq r$

$\Rightarrow$

$|s(l, m, n)-p(l, m, n)| \leq r$

$\forall l, m, n \in N$.

By equation (l) and definition of $p(l, m, n)$, we get

$\Rightarrow \quad s t-\lim \sup |p(l, m, n)-k|=0$

$\Rightarrow \quad s t-\lim p(l, m, n) \rightarrow^{r} k$.

Sufficiency: Because $s t-\lim p(l, m, n)=k$, we have

$\delta\left(\left\{(l, m, n) \in \boldsymbol{N}^{3}:|p(l, m, n)-k| \geq \epsilon\right\}\right)=0 \quad$ for each $\epsilon>0$.

It easy to see that the inclusion

$\left\{(l, m, n) \in N^{3}\right.$ :

$|p(l, m, n)-k| \geq \epsilon\} \supseteq\left\{(l, m, n) \in N^{3}:|s(l, m, n)-k| \geq r+\epsilon\right\}$ holds.

Because

$\delta\left(\left\{(l, m, n) \in \boldsymbol{N}^{3}:|p(l, m, n)-k| \geq \epsilon\right\}=0\right.$, we get $\left.\delta\left(\{l, m, n) \in N^{3}:|s(l, m, n)-k| \geq r+\epsilon\right\}\right)=0$.

Hence the theorem.

\section{THEOREM 3.9}

A triple sequence $s(l, m, n)$ is statistically analytic $\Leftrightarrow \exists$ a non-negative real number $r$ s.t. $s t-L I M^{r} s \neq \emptyset$.

PROOF

Since the triple sequence $S$ is statistically analytic, $\exists$ a positive real number $M$ s.t.

$$
\delta\left(\left\{(l, m, n) \in N^{3}:|(s(l, m, n))|^{1 / l+m+n} \geq M\right\}\right)=0
$$

Define

Then the set $s t-L I M^{r} s$ contains the origin of $\boldsymbol{R}$.

So we have $s t-L I M^{r} s \neq \emptyset$.

$k \in s t-L I M^{r} s$ 
i.e.,
$\delta\left(\left\{(l, m, n) \in N^{3}:|s(l, m, n)-k|^{1 / l+m+n} \geq r+\epsilon\right\}\right)=0$ for each $\epsilon>0$.

Then we say that almost all $s(l, m, n)$ are contained in some ball with any radius greater than $r$. So the triple sequence $s$ is statistically analytic.

\subsection{ALMOST CONVERGENCE OF TRIPLE SEQUENCE}

\section{DEFINITION 3.11}

A triple sequence $s(l, m, n)$ almost convergence to $L_{y}$ if for every $\epsilon>0, \exists N \in N$ s.t.

$\left|\frac{1}{p q r} \sum_{i=0}^{p-1} \sum_{j=0}^{q-1} \sum_{k=0}^{r-1} s(l+i, m+j, n+k)-L\right|<\epsilon$

$\forall p, q, r>N$ and all $(l, m, n) \in N \times N \times N$.

\section{DEFINITION 3.12}

The triple sequence $s(l, m, n)$ is almost Cauchy, if for every $\epsilon>0, \exists N \in N_{\text {s.t. }}$.

$\left|\begin{array}{c}\frac{1}{p_{1} q_{1} r_{1}} \sum_{i=0}^{p_{1}-1} \sum_{j=0}^{q_{1}-1} \sum_{k=0}^{r_{1}-1} s\left(l_{1}+i, m_{1}+j, n_{1}+k\right) \\ -\frac{1}{p_{2} q_{2} r_{2}} \sum_{i=0}^{p_{2}-1} \sum_{j=0}^{q_{2}-1} \sum_{k=0}^{r_{2}-1} s\left(l_{2}+i, m_{2}+j, n_{2}+k\right)\end{array}\right|<\epsilon$

$\forall p_{1}, q_{1}, r_{1}, p_{2}, q_{2}, r_{2}>N$ and all

$\left(l_{1}, m_{1}, n_{1}\right),\left(l_{2}, m_{2}, n_{2}\right) \in \boldsymbol{N} \times \boldsymbol{N} \times \boldsymbol{N}$.

\section{LEMMA 3.1}

The triple sequence $(s(l, m, n))$ is almost convergent $\Leftrightarrow$ it is almost Cauchy.

\section{PROOF}

Suppose the triple sequence $(s(l, m, n))$ is almost convergent to $\mathrm{L}$.

Then, for every $\epsilon>0, \exists N \in N_{\text {s.t. }}$.

$\left|\frac{1}{p q r} \sum_{i=0}^{p-1} \sum_{j=0}^{q-1} \sum_{k=0}^{r-1} s(l+i, m+j, n+k)-L\right|<\epsilon$

$\forall p, q, r>N$ and all $(l, m, n) \in N \times N \times N$

$$
\begin{aligned}
& \therefore\left|\begin{array}{l}
\frac{1}{p_{1} q_{1} r_{1}} \sum_{i=0}^{p_{1}-1} \sum_{j=0}^{q_{1}-1} \sum_{k=0}^{q_{1}-1} s\left(l_{1}+i, m_{1}+j, n_{1}+k\right) \\
-\frac{1}{p_{2} q_{2} r_{2}} \sum_{i=0}^{p_{2}-1} \sum_{j=0}^{q_{2}-1} \sum_{k=0}^{r_{2}-1} s\left(l_{2}+i, m_{2}+j, n_{2}+k\right)
\end{array}\right| \\
& \leq\left|\frac{1}{p_{1} q_{1} r_{1}} \sum_{i=0}^{p_{1}-1} \sum_{j=0}^{q_{1}-1} \sum_{k=0}^{r_{1}-1} s\left(l_{1}+i, m_{1}+j, n_{1}+k\right)-L\right| \\
& +\left|\frac{1}{p_{2} q_{2} r_{2}} \sum_{i=0}^{p_{2}-1} \sum_{j=0}^{q_{2}-1} \sum_{k=0}^{r_{2}-1} s\left(l_{2}+i, m_{2}+j, n_{2}+k\right)-L\right| \\
& <\frac{E}{2}+\frac{E}{2}=\epsilon \\
& \forall p_{1}, q_{1}, r_{1}, p_{2}, q_{2}, r_{2}>N \text { and all } \\
& \left(l_{1}, m_{1}, n_{1}\right),\left(l_{2}, m_{2}, n_{2}\right) \in \mathbf{N} \times \mathbf{N} \times \mathbf{N} \text {. }
\end{aligned}
$$

Hence the triple sequence $s(l, m, n)$ is almost Cauchy.

Now, suppose that the triple sequence $(s(l, m, n))$ is almost Cauchy.

Then, for every $\epsilon>0, \exists N \in N$ s.t.

$\left|\begin{array}{l}\frac{1}{p_{1} q_{1} r_{1}} \sum_{i=0}^{p_{1}-1} \sum_{j=0}^{q_{1}-1} \sum_{k=0}^{r_{1}-1} s\left(l_{1}+i, m_{1}+j, n_{1}+k\right) \\ -\frac{1}{p_{2} q_{2} r_{2}} \sum_{i=0}^{p_{2}-1} \sum_{j=0}^{q_{2}-1} \sum_{k=0}^{r_{2}-1} s\left(l_{2}+i, m_{2}+j, n_{2}+k\right)\end{array}\right|<\frac{\epsilon}{2}$

$\forall p_{1}, q_{1}, r_{1}, p_{2}, q_{2}, r_{2}>N$ and all $\left(l_{1}, m_{1}, n_{1}\right),\left(l_{2}, m_{2}, n_{2}\right) \in N \times N \times N$.

Taking $l_{1}=l_{2}=l_{0}, m_{1}=m_{2}=m_{0}$ and $n_{1}=n_{2}=n_{0}$ in relation $(1)$, we obtain that

$\left(\frac{1}{p q r} \sum_{i=0}^{p-1} \sum_{j=0}^{q-1} \sum_{k=0}^{r-1} s\left(l_{1}+i, m_{1}+j, n_{1}+k\right)\right)_{p, q_{2} r=1}^{\infty}$

is a Cauchy sequence in $\boldsymbol{C}$. Therefore the set of complex numbers is convergent since $\boldsymbol{C}$ is complete.

Let

$P-\lim _{p, q, r \rightarrow \infty} \frac{1}{\text { p q } r} \sum_{i=0}^{p-1} \sum_{j=0}^{q-1} \sum_{k=0}^{r-1} s\left(l_{1}+i, m_{1}+j, n_{1}+k\right)=L$ Then for every $\epsilon>0, \exists N_{1} \in N$ s.t.

$\left|\frac{1}{p q r} \sum_{i=0}^{p-1} \sum_{j=0}^{q-1} \sum_{k=0}^{r-1} s\left(l_{1}+i, m_{1}+j, n_{1}+k\right)-L\right|<\frac{\epsilon}{2}$ $\forall p, q, r>N_{1}$.

It follows that, 
$\left|\frac{1}{p \quad q r} \sum_{i=0}^{p-1} \sum_{j=0}^{q-1} \sum_{k=0}^{r-1} s(l+i, m+j, n+k)-L\right|$

$\leq\left|\begin{array}{c}\frac{1}{p q r} \sum_{i=0}^{p-1} \sum_{j=0}^{q-1} \sum_{k=0}^{r-1} s(l+i, m+j, n+k) \\ -\frac{1}{p q r} \sum_{i=0}^{p-1} \sum_{j=0}^{q-1} \sum_{k=0}^{r-1} s\left(l_{1}+i, m_{1}+j, n_{1}+k\right)\end{array}\right|$

$+\left|\frac{1}{p q r} \sum_{i=0}^{p-1} \sum_{j=0}^{q-1} \sum_{k=0}^{r-1} s\left(l_{1}+i, m_{1}+j, n_{1}+k\right)-L\right|$

$<\frac{E}{2}+\frac{E}{2}=\epsilon$

$\forall p, q, r>\max \left(N, N_{1}\right)$ and $\mathrm{V}$

$(l, m, n) \in \boldsymbol{N} \times \boldsymbol{N} \times \boldsymbol{N}$.

So, the triple sequence $s(l, m, n)$ is almost convergent to $L$.

This completes the proof.

\section{CONCLUSION}

In this paper, we have established triple sequences using single sequences and double sequences. This extension can be used to verify Cauchy triple sequence, triple subsequence and convergence (or) divergence for a given sequence. Also we have to seen that rough statistical convergence on triple sequence and almost convergence of triple sequence.

Further we have discussed the study of the basic properties of triple sequence. This study can be extended in future for many sequences (four, five and so on).

\section{REFERENCE}

[1] Apostol T.M, Mathematical Analysis, Second edition, Addison Wesley, 1974.

[2] Ayhan Esi, Necdet Catalbas.M "Almost convergence of triple sequences", Global Journal of sequences", Mathematical Analysis, 2 (1) (2014), 6-10.

[3] Ahmet Sahiner, Mehmet Gurdal and Kadriye Duden.F, "Triple sequences and their statistical convergence" Selcuk Journal of Applied Mathematics,Vol.8.No.2. (2007), 49-55.

[4] Bartle R.G and Sherbert D.R, Introduction to Real Analysis, Second edition, Wiley and Sons, $1992 .$.

[5] Chen W.W.L, Fundamentals of Analysis, Published by W.W.L. Chen via Internet, 2003.
[6] Cunjalo.F "Almost convergence of double sequencesSome Analogies between measure and category", Math. Maced., 5(2007), 21-24.

[7] Debnath.S and Das.B.C., Regular matrix transformation on triple sequence spaces. Bol. Soc. Paran. Mat. 34(2) (2016), $1-12$.

[8] Gupta S.L and N.Rani, Priciples of real Analysis, Vikas Publishing House, New Delhi,1998.

[9] Hamilton.H.J: Transformations of multiple sequences. Duke Math. Jour.2 (1936), 29-60.

[10] Lorentz G.G," A contribution to the principles of divergent sequences", Acta. Math.,80(1948), 167-190.

[11] Mishra.V.N Khatri.K and Mishra.L.N : Using linear operators to approximate singnals of Lip of $(\alpha, \mathrm{p})$, ( $\mathrm{p} \geq 1$ )- class . Filomat.27(2) (2013), 355-365.

[12] Pringsheim A, "Zur Theori der zweifach unendlichen Zahlenfolgen", Math Ann.,53(1900), 289-321.

[13] Rudin W, Priciples of Mathematical Analysis, Third edition, McGRAW-Hill, 1976.

[14] Stromberg K.R, A Introduction to Classical Real Analysis, Wadsworth, 1981.

[15] Shyamal Debnath, Subramanian.N, "Rough statistical convergence on triple sequences", Proyecciones Journal of Mathematics, Vol. 36, No.4, (2017), 685-699. 